Original

\title{
Meteorological influences on the incidence of lichen planus in a north Indian population
}

\author{
Ravi Prakash S. Mohan, Soumi Ghanta, Sankalp Verma, Neha Agarwal, \\ Nupur Gupta, and Udita Singh
}

Department of Oral Medicine and Radiology, Kothiwal Dental College Research Centre and Hospital, India

(Received March 22, 2013; Accepted September 27, 2013)

\begin{abstract}
Lichen planus is a chronic, autoimmune, mucocutaneous disease that shows differences in clinical presentation at different times of the year. The present retrospective study was conducted to clarify the meteorological factors that influence the incidence of lichen planus, as well as the general features and clinical presentation of this condition during three consecutive years, 2008, 2009, and 2010, in Moradabad district (Western Uttar Pradesh, India). The study group comprised 1,355 patients extracted from the records of the outpatient department of Kothiwal Dental College Research Centre and Hospital, who were clinically diagnosed as having lichen planus during this three-year period. The highest number of patients (735) were recorded in summer, and the lowest (56) in winter. Females were affected more often, and psychosocial influences were also evident. There were significant differences in the incidence of lichen planus in different seasons, and the summer peak was attributable to the intensity of sun exposure (actinic lichen planus and summertime lichen planus). (J Oral Sci 55, 311-318, 2013)
\end{abstract}

Keywords: lichen planus; meteorology; actinic lichen planus.

Correspondence to Dr. Ravi Prakash Sasankoti Mohan, Department of Oral Medicine and Radiology, Kothiwal Dental College Research Centre and Hospital, Mora Mustaqueem, Kanth Road, Moradabad, Uttar Pradesh 244401, India

E-mail: sasan_ravi@rediffmail.com

doi.org/10.2334/josnusd.55.311

DN/JST.JSTAGE/josnusd/55.311
Introduction
It is generally agreed that the patterns of skin diseases
differ among countries, and also within various regions
of a country, depending on environmental, social,
economic, or racial factors (1). Skin diseases tend to have
low mortality but high morbidity (1), and their prevalence
is affected by climate (2), which has an important influ-
ence on human health and general well-being (3). It has
been generally observed that the patterns of skin diseases
in patients presenting at dermatology clinics differ at
various times throughout the year, especially in tropical
and subtropical countries (2). India is a subtropical
country where changes in climate have a definite impact
on the incidence of skin diseases.

Lichen planus is a chronic, autoimmune, mucocutaneous, psychosocial disease that can affect the oral mucosa, skin, genital mucosa, scalp and nails $(4,5)$. The disease has most often been reported in middleaged patients, being more common in females than in males (4-6). It is also seen in children, although rarely. Clinically, it can present as white striations (Wickham's striae), white papules, white plaque, erythema, erosion or blisters (5). Oral lichen planus is typically present in the posterior buccal mucosa, on the tongue, or on the alveolar ridge/gingiva, but rarely on the palate or lip vermillion $(4,6,7)$. There are six clinical variants: reticular, papular, plaque-like, erosive, atrophic, and bullous $(4,5)$, and some can co-exist in the same patient. Lichen planus is a T-cell-mediated autoimmune disease in which autocytotoxic $\mathrm{CD} 8^{+} \mathrm{T}$-cells trigger apoptosis of epithelial cells. Microscopically, the disease is characterized by a dense subepithelial lympho-histocytic infiltrate, increased numbers of intra-epithelial lymphocytes, and degeneration of basal keratinocytes, which form colloid 
or civatte bodies (5).

One variant of lichen planus is noticeably affected by climate in tropical and semitropical countries. This variant arises preferentially on exposed parts of the body, mainly the face, especially on the forehead, cheeks and lips, and also the upper area of the chest, the dorsum of the hands, and lower extensor surface of the forearms. This condition is more prevalent during the summer months, late spring and early autumn, i.e., when the light and sun are more intense. Itching or discomfort is minimal or absent during the winter (8).

The incidence of lichen planus has been increasing, and its treatment is challenging for dermatologists as well as dentists, as it tends to be chronic and recalcitrant to therapy. Relapses are common even after remission following treatment (9).

The aim of this retrospective study was to clarify the meteorological factors influencing the incidence of lichen planus, as well as the general features and clinical presentation of this lesion, and the effects of socioeconomic factors, stress and some systemic illnesses on its incidence during a three-year period in 2008, 2009, and 2010 in Moradabad district (Western Uttar Pradesh, India).

\section{Materials and Methods}

The study group comprised 1,355 patients whose data were extracted from the records of the outpatient department of Kothiwal Dental College Research Centre and Hospital, who had been clinically diagnosed as having lichen planus in 2008, 2009, and 2010. Data related to patient age, sex, socio-economic condition, signs and symptoms, and treatment outcome were extracted from the case history records. Patients from both urban and rural areas were taken into consideration.

Meteorological data for the study period were obtained from the Government Department of Meteorology for the Moradabad region.

\section{Clinical diagnostic criteria for lichen planus}

The diagnostic criteria for lichen planus may have changed over time in the light of increased knowledge. The clinical diagnostic criteria for oral lesions used in this study were as follows (4):

1. The presence of keratotic, pinhead-sized, white, slightly elevated papules (papular lichen planus), which may be discrete or arranged in reticular (reticular lichen planus) or plaque-like (plaque-like lichen planus) configurations

2. Atrophic lichen planus, characterized by thinning of the epithelium leading to the appearance of atrophic red areas within the white lesions

3. Erosive (ulcerative) lichen planus, characterized by areas of well-defined ulceration within the abovementioned lesions

4. Bullous lichen planus, characterized by the presence or development of bullous areas within the abovementioned lesions

The clinical diagnostic criteria for skin lesions used in this study were as follows $(8,10)$ :

1. Hypertrophic lichen planus, characterized by thickened, hyperkeratotic, intensely pruritic lesions ranging in color from purple to gray or black

2. Annular-type lichen planus, characterized by erythematous brownish plaques with an annular configuration

3. Actinic-type lichen planus, characterized by hyperpigmented or violaceous to blue-brown papules with a thready, rolled edge and well defined borders with minimal scaling on exposed parts of the body (such as, forehead, face, nape of neck, hand etc.) and also a history of onset especially in the spring and summer

4. Miscellaneous types of lichen planus, including classic lichen planus present elsewhere on the body or mucous membrane, follicular spinous lesions, cicatricial alopecia of the scalp and non-scarring alopecia of the axilla and groin, or nail changes showing longitudinal ridging and grooving, splitting (onchyoschizia), longitudinal striation (onychorrhexis), anonychia and subungual hyperkeratosis

Histopathological diagnosis was performed whenever there was any doubt about the clinical diagnosis.

\section{Inclusion criteria}

All diagnosed patients with lichen planus, both adults and children, were included in this study.

\section{Exclusion criteria}

Patients with clinicopathological signs of epithelial dysplasia, or use of any drugs or dental restorations implicated in lichenoid reactions, were excluded from the study.

The study protocol was reviewed and approved by the Research Ethics Committee of M.J.P. Rohilkhand University, Uttar Pradesh (KDCRC/ETH/OMDR/2011/03).

\section{Results}

Hospital records of Kothiwal Dental College for the years 2008, 2009 and 2010 were explored. A total of 1,355 patients with a confirmed diagnosis of lichen planus were extracted and included. A total of 415 cases were recorded in 2008, 420 in 2009, and 520 in 2010. 




Fig. 1 Year-wise distribution of cases across different seasons. In all of the three studied years, most cases were reported during summer, while the least were reported during winter $(P=0.118)$.

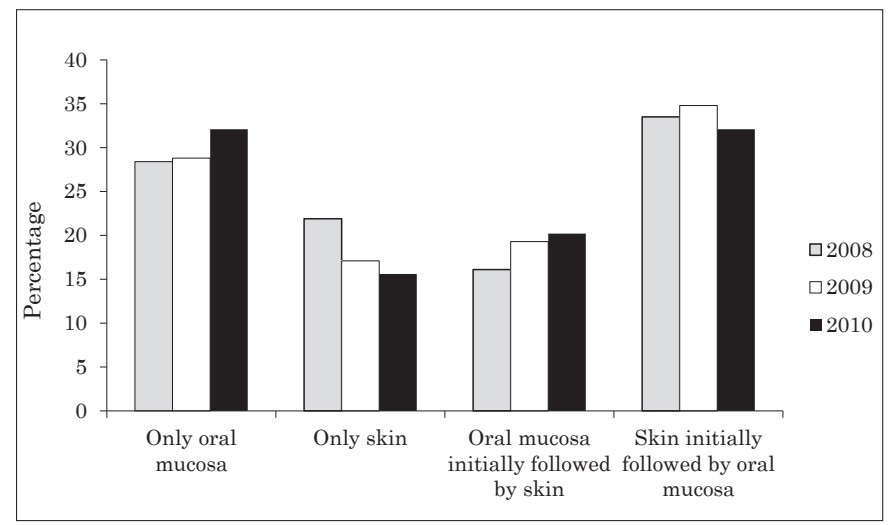

Fig. 3 Distribution of cases according to area of involvement. The majority of cases in each year involved the skin initially, followed by the oral mucosa; the next most common type was involvement of the oral mucosa alone. Involvement of the skin alone accounted for the lowest number of patients, except in 2008 $(P=0.154)$.

Year-wise distribution of cases across different seasons In all of the three years, the maximum number of cases was reported during summer, whereas the minimum number was reported during winter. The pre-monsoon and post-monsoon seasons showed relatively higher proportions of patients than in winter, but the numbers were much lower than those during summer. Statistically, there were no significant differences in the seasonal distribution of patients among the three groups (groups for 2008, 2009, and 2010) $(P=0.118)$ (Fig. 1).

\section{Nature of cases (new/old) in different seasons}

In all of the three years investigated, the proportional distribution of new and old cases varied significantly across different seasons. There was a significant rise in the proportion of new cases during the pre-monsoon and



Fig. 2 Nature of cases (new/old) in different seasons during the entire three-year period. A significant increase in the proportion of new cases was seen during the pre-monsoon and summer seasons, as compared with the post-monsoon and winter seasone, in all three of the studied years. Both old and new cases showed the lowest occurrence in winter.

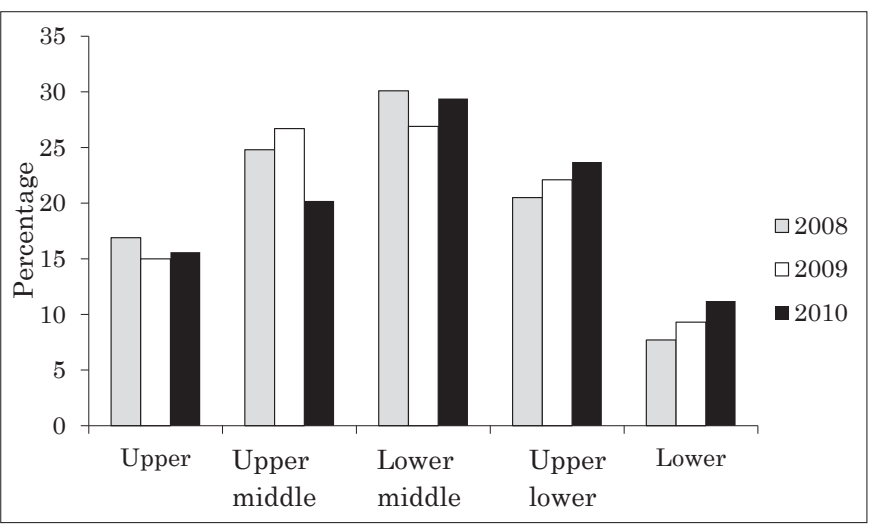

Fig. 4 Distribution of cases according to socio-economic status. The majority of patients, irrespective of the year, were from the upper middle and lower middle socioeconomic strata $(P=0.281)$ [Kuppuswamy's socio-economic status scale was used].

summer seasons relative to the post-monsoon and winter seasons (Fig 2). July saw the highest number of cases and January the lowest.

A total of $508(37.5 \%)$ patients were males and 847 $(62.5 \%)$ were females, the latter outnumbering the former by about $2: 1$.

\section{Distribution of cases according to area of involvement}

We divided the cases into four categories according to the area of involvement: oral mucosa only, skin only, oral mucosa initially followed by the skin, and skin initially followed by the oral mucosa. The latter accounted for the highest number of cases in each year, followed by involvement of the oral mucosa only; involvement of the skin only accounted for the lowest number of cases, except in 2008, where involvement of the oral mucosa 


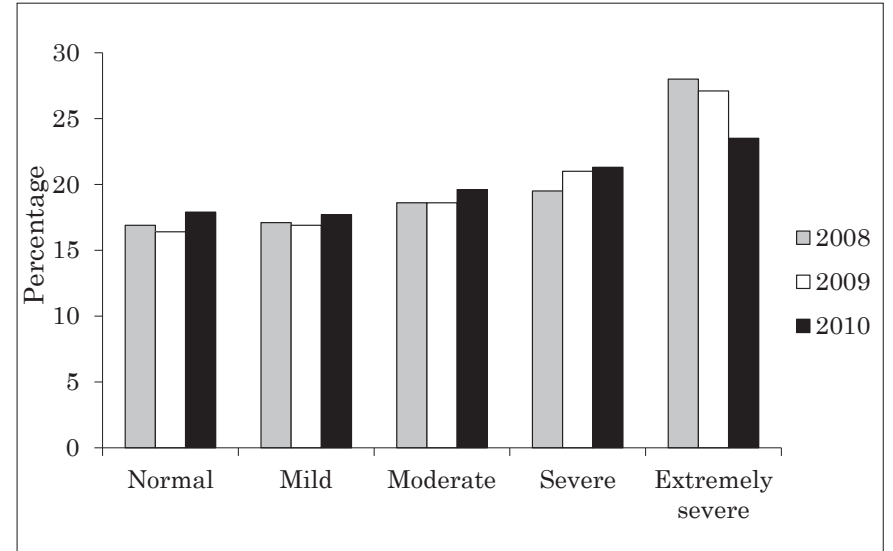

Fig. 5 Distribution of cases during the three-year study period in relation to stress. Most patients with lichen planus were under extreme stress [Depression-Anxiety-Stress Scale (DASS) was used].

initially followed by the skin accounted for the lowest number. Statistically, these differences were not significant $(P=0.154)$ (Fig. 3).

\section{Distribution of cases according to socio-economic status}

According to Kuppuswamy's socio-economic status scale, we divided the cases into five socio-economic groups: upper class, upper middle class, lower middle class, upper lower class, and lower class. We found that the majority of cases, irrespective of the year, were from the upper middle and lower middle socio-economic classes. Again, among these two classes, the lower middle was affected more than the upper middle class. Statistically, there was no significant difference among the three years with respect to the socio-economic strata of the patients $(P=0.281)$ (Fig. 4$)$.

\section{Distribution of cases during the three years according to stress condition}

We divided the total patients into five stress conditions using the Depression-Anxiety-Stress Scale (DASS): normal, mild, moderate, severe, and extremely severe. Less than one-fifth (16.4\% to $17.9 \%)$ of the patients had stress levels within the normal range. Most of the lichen planus patients were under extremely severe stress. Statistically, there were no significant differences in stress condition throughout the three studied years $(P$ $=0.928)$ (Fig. 5).

\section{Distribution of cases in relation to history of systemic illness}

We took into consideration some systemic diseases, including diabetes, hypertension, hepatitis, malignancy,

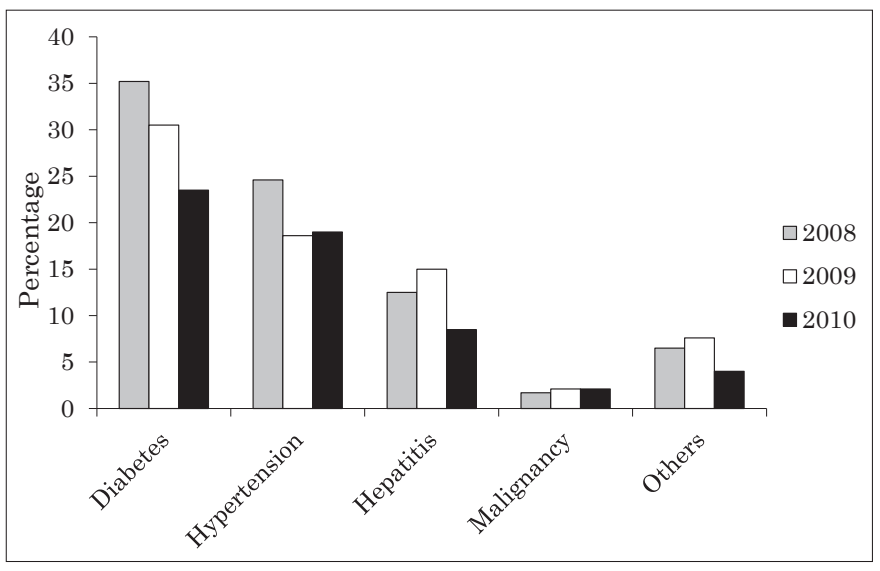

Fig. 6 Distribution of patients with a history of systemic illness. Diabetes was the most common presenting systemic illness, followed by hypertension and hepatitis.

Table 1 Distribution of different types of lichen planus

\begin{tabular}{clcc}
\hline SN & Clinical types of lichen planus & No. of cases & Percentage (\%) \\
\hline 1 & Reticular & 406 & 30 \\
2 & Annular & 68 & 5 \\
3 & Papular & 81 & 6 \\
4 & Erosive & 203 & 15 \\
5 & Plaque-like & 108 & 8 \\
6 & Atrophic & 54 & 4 \\
7 & Bullous & 41 & 3 \\
8 & Actinic & 285 & 21 \\
9 & Hypertrophic & 68 & 5 \\
10 & Miscellaneous & 41 & 3 \\
& & 1,355 & 100 \\
\hline
\end{tabular}

During the study period, the majority of cases were the reticular type, followed by the actinic type and erosive type.

and others (gastrointestinal diseases, alopecia areata, dermatitis herpetiformis, Hashimoto's thyroiditis, pemphigus foliaceus, pemphigus vulgaris, systemic sclerosis, myasthenia gravis etc.).

In all of the three studied years, diabetes was the most common presenting systemic illness, followed by hypertension and hepatitis. During the study period, $1.7-2.1 \%$ of the patients had malignancy and 4-6.5\% had other systemic illnesses (Fig. 6).

\section{Distribution of different types of lichen planus}

Among the 1,355 patients, most (30\%) were diagnosed as having reticular-type lichen planus, followed by the actinic type (21\%) and erosive type (15\%) (Table 1).

\section{Distribution of different types of lichen planus across different seasons}

A total of nine major types and 8-9 less frequent types 
Table 2 Distribution of different types of lichen planus across different seasons

\begin{tabular}{|c|c|c|c|c|c|c|c|c|c|c|c|}
\hline \multirow[b]{3}{*}{ SN } & \multirow{3}{*}{$\begin{array}{l}\text { Clinical types of } \\
\text { Lichen Planus }\end{array}$} & \multicolumn{8}{|c|}{ Seasons } & \multirow{2}{*}{\multicolumn{2}{|c|}{$\begin{array}{c}\text { Statistical } \\
\text { significance }\end{array}$}} \\
\hline & & \multicolumn{2}{|c|}{$\begin{array}{c}\text { Winter } \\
(n=56)\end{array}$} & \multicolumn{2}{|c|}{$\begin{array}{l}\text { Pre-monsoon } \\
\quad(n=334)\end{array}$} & \multicolumn{2}{|c|}{$\begin{array}{c}\text { Summer } \\
(n=735)\end{array}$} & \multicolumn{2}{|c|}{$\begin{array}{l}\text { Post-monsoon } \\
\quad(n=230)\end{array}$} & & \\
\hline & & No. & $\%$ & No. & $\%$ & No. & $\%$ & No. & $\%$ & $\chi^{2}$ & $P$ \\
\hline 1 & Reticular & 20 & 35.7 & 102 & 30.5 & 186 & 25.3 & 98 & 42.6 & 26.058 & $<0.001$ \\
\hline 2 & Annular & 5 & 8.9 & 12 & 3.6 & 42 & 5.7 & 9 & 3.9 & 4.557 & 0.207 \\
\hline 3 & Papular & 6 & 10.7 & 19 & 5.7 & 32 & 4.4 & 24 & 10.4 & 13.863 & 0.003 \\
\hline 4 & Erosive & 9 & 16.1 & 54 & 16.2 & 101 & 13.7 & 39 & 17.0 & 2.013 & 0.570 \\
\hline 5 & Plaque-like & 4 & 7.1 & 20 & 6.0 & 72 & 9.8 & 12 & 5.2 & 7.557 & 0.057 \\
\hline 6 & Atrophic & 3 & 5.4 & 15 & 4.5 & 27 & 3.7 & 9 & 3.9 & 0.689 & 0.876 \\
\hline 7 & Bullous & 2 & 3.6 & 10 & 3.0 & 21 & 2.9 & 8 & 3.5 & 0.290 & 0.962 \\
\hline 8 & Actinic & 3 & 5.4 & 81 & 24.3 & 183 & 24.9 & 18 & 7.8 & 41.132 & $<0.001$ \\
\hline 9 & Hypertrophic & 2 & 3.6 & 16 & 4.8 & 44 & 6.0 & 6 & 2.6 & 4.529 & 0.210 \\
\hline 10 & Miscellaneous & 2 & 3.6 & 5 & 1.5 & 27 & 3.7 & 7 & 3.0 & 3.768 & 0.288 \\
\hline
\end{tabular}

The reticular type was most common in the post-monsoon season and least common during the summer. The actinic type was more common in the pre-monsoon and summer seasons than in the winter and post-monsoon seasons $(P<0.001)$.

Table 3 Most common recalcitrant types of lichen planus

\begin{tabular}{clrccc}
\hline & & \multicolumn{2}{c}{ New cases $(n=780)$} & \multicolumn{2}{c}{ Old cases $(n=575)$} \\
SN & Clinical types of lichen planus & No. & $\%$ & No. & $\%$ \\
\hline 1 & Reticular & 333 & 42.7 & 73 & 12.7 \\
2 & Annular & 50 & 6.4 & 18 & 3.1 \\
3 & Papular & 46 & 5.9 & 35 & 6.1 \\
4 & Erosive & 70 & 9 & 133 & 23.1 \\
5 & Plaque-like & 44 & 5.6 & 64 & 11.1 \\
6 & Atrophic & 8 & 1 & 46 & 8 \\
7 & Bullous & 26 & 3.3 & 15 & 2.6 \\
8 & Actinic & 160 & 20.5 & 125 & 21.7 \\
9 & Hypertrophic & 14 & 1.8 & 54 & 9.4 \\
10 & Miscellaneous & 29 & 3.7 & 12 & 2.1 \\
\hline
\end{tabular}

Whereas most new cases were diagnosed as the reticular type, the majority of old cases reported as exacerbations were diagnosed as the erosive type, followed by the actinic type.

of lichen planus categorized as miscellaneous were encountered in our study. Seasonal variability in the distribution of the reticular, papular and actinic types was observed. The reticular type was most frequent in the post-monsoon season (42.6\%) and least frequent during summer $(25.3 \%)$, showing a significant seasonal variability $(P<0.001)$. The papular type was more frequent in the post-monsoon $(10.4 \%)$ and winter $(10.7 \%)$ seasons than in the pre-monsoon $(5.7 \%)$ and summer $(4.4 \%)$ seasons, the seasonal variability being significant $(P=$ $0.003)$. Actinic-type lichen planus was more frequent in the pre-monsoon $(24.3 \%)$ and summer $(24.9 \%)$ seasons than in the winter $(5.4 \%)$ and post-monsoon $(7.8 \%)$ seasons $(P<0.001)$ (Table 2$)$.

\section{Most common recalcitrant type of lichen planus}

Most $(23.1 \%)$ cases of recalcitrant lichen planus were the erosive type, followed by the actinic type (21.7\%), whereas most new cases were the reticular type (Table 3).

\section{Discussion}

The prevalence of skin diseases in a community is affected by many factors, but climate plays a major role (2). The Meteorological Department in India was established as a national agency in 1875 , amalgamating various provincial meteorological services. Basically, the climate of India is dominated by the summer monsoon. According to the India Meteorology Department, the year can be divided into four seasons (11, http://www. imdpune.gov.in/research/ncc/climatebulletin/):

i) Winter (January and February)

ii) Pre-monsoon or hot weather season (MarchApril-May)

iii) Southwest or summer monsoon season (JuneJuly-August-September)

iv) Post-monsoon season (October-November- 


\section{December)}

Lichen planus is a common papulosquamous disease (10), and can affect either the skin, mucosa, or both (12). In our present study, in all of the three years investigated, most cases were reported during summer, and the least during winter. These findings are in accordance with those of previous studies by el-Zawahry (8), Boyd and Neldner (10), Ramirez et al. (13), and Issacson et al. (14), who all suggested that the condition is more prevalent during the summer months, the end of the pre-monsoon, and the early post-monsoon, when sunlight is more intense. el-Zawahry (8) demonstrated that exposure to sunlight increases the itching and the severity of the disease, and that protection from sunlight lessens the discomfort. They designated this type of lichen planus to be "lichen planus tropicus" rather than "lichen planus actinicus", because its development is linked to not only sunlight but also a tropical or semitropical climate. Kim and Mikkilineni (15) suggested that ultraviolet radiation was an important factor, possibly leading to the expression of altered selfantigens on basal keratinocytes, allowing the recruitment of cytotoxic T-cells, which play a role in development of the lesions.

Oral lichen planus is the most commonly reported form after the cutaneous type. Up to $65 \%$ of patients with classical cutaneous lichen planus have oral involvement (10). The cutaneous lesions are pruritic, mainly annular in form, and intensely pigmented, being erythematous to violaceous $(8,10)$. Brownish and dark long-standing post-lichen pigmentation remains after resolution of the lesions, and this may be annoying to patients if it affects exposed parts of the body (8). In all of the three years we studied, the proportional distribution of new and old cases varied significantly across different seasons. There was a significant rise in the proportion of new cases during the pre-monsoon and summer seasons relative to the post-monsoon and winter seasons. Also, a higher number of recalcitrant cases than new cases were observed in the winter and post-monsoon seasons. The intensity of sun exposure is the probable reason for the greater number of new cases during summer and the premonsoon and the lower number in winter. el-Zawahry (8) demonstrated that the summer months have a detrimental effect on lesions, and that most patients seen during winter complain primarily about residual pigmentation.

In all of the three years we studied, there was a gradual increase in the number of cases from January to July, and the peak incidence occurred in July, in accordance with the findings of Boyd and Neldner (10). Those authors referred to a variety of lichen planus named actinic lichen planus which has been given many names in the ensuing years, including lichen planus subtropicus, lichen planus tropicus, summertime actinic lichenoid eruption, lichen planus actinicus, lichen planus atrophicus annularis and lichenoid melanodermatosis in which sunlight plays an important role, the majority of cases occurring in summer and spring.

In our present series, the majority of patients were female, being concordant with the studies of Ingafou et al. (4), Mollaoglu (12), Scully and Carrozzo (16), Rodríguez-Núñez et al. (17), and Wolff $\mathrm{K}$ et al. (18). Rodríguez-Núñez et al. (17) and Lacy et al. (19) suggested that males tend to be mostly affected in their early thirties, whereas most women develop lichen planus in their fifties. However, el-Zawahry (8) considered actinic lichen planus to be more common in males than in females.

In each of the studied years, lichen planus involving the skin initially, followed by the oral mucosa, accounted for the majority of cases, followed by cases involving the oral mucosa alone, whereas cases involving only the skin were the least common, except in 2008. Boyd and Neldner (10) suggested that cases involving only the oral mucosa accounted for $15-35 \%$. They also referred to a Scandinavian study suggesting that oral involvement was eight times more common than cutaneous involvement. They also suggested that $20-34 \%$ of patients with oral lichen planus also have concomitant cutaneous involvement, or that conversely, up to $65 \%$ of patients with classical cutaneous lichen planus have oral involvement. el-Zawahry (8) considered that males are more prone to lichen planus tropicus, where the exposed parts of the body, mainly the face, and especially the forehead, cheeks and lips, together with the upper area of the chest, the dorsum of the hands, and the lower parts of the extensor surface of the forearms, are mainly affected.

We found that the majority of patients, irrespective of the year, were from the upper middle and lower middle socio-economic classes. This accords with the study of Ingafou et al. (4), who concluded that there was an unequal distribution of patients across the socio-economic groups, ranging from $2.3 \%$ of employers or managers to $20 \%$ of semi-skilled manual workers.

We found that most patients with lichen planus, including both cutaneous and oral lesions, were under extreme stress, in agreement with the study of Girardi et al. (20), suggested an association between oral lichen planus and anxiety, salivary levels of dehydroepiandrosterone (DHEA) and cortisol playing an important role. On the other hand, Allen et al. (21) suggested that patients with oral lichen planus had no greater tendency for anxiety or stressful life events than other individuals.

Lichen planus has been reported to be associated with 
a variety of totally unrelated disorders. Although it is very difficult to confirm such an association, it seems that immunodeficiency would likely be the underlying cause of both conditions in some cases (10). In the present series, we found that diabetes was the most common associated systemic illness, followed by hypertension and hepatitis. Defective carbohydrate metabolism is probably responsible for the association between lichen planus and diabetes. Boyd and Neldner (10) and Christensen et al. (22) cited the same study by Lynch that had demonstrated a positive correlation between lichen planus and high blood pressure. The triad of hypertension, diabetes mellitus and lichen planus has been reported as Grinspan's syndrome (10). Boyd and Neldner (10) reported that the incidence of hepatitis in patients with lichen planus varied from $9.5 \%$ to $13.5 \%$. Strauss et al. (23) suggested that the most common liver ailments associated with lichen planus are chronic active hepatitis and primary biliary cirrhosis, and also proposed that administration of D-penicillamine may predispose a patient with primary biliary cirrhosis to the development of lichen planus as well as aggravating any existing disease. Al-Khenaizan (24) reported a case of lichen planus occurring after hepatitis B vaccination. They suggested that a chronic graft-versus-host-like autoimmune reaction might have been responsible for the pathogenesis, involving possible cross-reactivity between HBsAg use in HBV vaccines and shared epitopes on keratinocytes. Bagán et al. (25) have concluded that there is an association between hepatitis $\mathrm{C}$ virus infection and oral lichen planus. In our present series, a small proportion of the patients had associated malignancy. Boyd and Neldner (10) demonstrated that lichen planus pemphigoides was associated with stomach cancer, lymphosarcoma, reticulum cell sarcoma, neuroblastoma, craniopharyngioma and pararenal malignancy. They also reported one case of pituitary adenoma in a patient with bullous lichen planus, and cited a study by Goihman-Yahr et al. in which a case of lichen planus coincided temporally with malignant fibrohistiocytoma.

In the present study, we divided the cases into ten clinical variants of lichen planus: reticular, plaque-like, papular, atrophic, erosive, bullous, actinic, hypertrophic, annular, and miscellaneous. Patients who had lichen planus planopillaris had classic lichen planus lesions elsewhere on the body or mucous membrane, follicular spinous lesions, cicatricial alopecia of the scalp and nonscarring alopecia of the axilla and groin, and those who had nail lichen planus showed longitudinal ridging and grooving, splitting (onchyoschizia), longitudinal striation (onychorrhexis), anonychia, and subungual hyperkeratosis; these two types were included in the miscellaneous group. We found that the reticular lichen planus was the most common type, followed by the actinic and erosive types, in agreement with the studies of Boyd and Neldner (10) and Ingafou et al. (4). We found that all patients in the miscellaneous group had Graham-Little-PiccardiLassueur syndrome, characterized by cicatricial alopecia of the scalp and non-scarring alopecia of the axilla and groin, follicular spinous papules on the body, scalp or both, and pterygium formation on the nails, associated with oral lichen planus.

We found that reticular-type lichen planus showed the highest prevalence in the post-monsoon season, and was least common during summer, whereas the actinic type was more common in the pre-monsoon and summer seasons than in the winter and post-monsoon seasons.

Among recalcitrant types of lichen planus, the erosive type was the most common, followed by the actinic type. The erosive type is most resistant to treatment. el-Zawahry (8) reported a female patient who had a history of recurrence each summer over an 8-year period, exhibiting involution of the lesions during winter, in accord with our findings. Most of the new cases were the reticular type, whereas old and recurrent cases were generally the erosive type, which may account for the fact that reticular lichen planus often changes into atrophic or erosive variants in the chronic phase. The literature refers little to this aspect.

As India is a subtropical $\left(6^{\circ} 45^{\prime}-37^{\circ} 6^{\prime} \mathrm{N}, 68^{\circ} 7^{\prime}-97^{\circ} 5^{\prime} \mathrm{E}\right)$ country, the high temperature and humidity throughout the year appear to account for the large number of patients presenting with lichen planus, especially in the summer.

Most patients with actinic lichen planus presented with skin lesions alone. Sunlight plays an important role in the pathogenesis of actinic lichen planus by altering the expression of self-antigen on basal keratinocytes and recruitment of cytotoxic T-cells.

Moreover, stress, especially in women as a result of cultural factors (a lifestyle confined to the family and home, and lack of social activities), and also unemployment in men, is an important reason for the large number of patients with lichen planus in India.

There is definite correlation between meteorological influence and the occurrence and remission of lichen planus, especially in tropical and semitropical countries, but a clear linear correlation between meteorological parameters and the disease presentation pattern could not be established in the present study. Further investigations will be required to clarify whether such a correlation exists. 


\section{References}

1. Sarkar SK, Islam AKMS, Sen KG, Ahmed ARS (2010) Pattern of skin diseases in patients attending OPD of Dermatology Department at Faridpur Medical College Hospital, Bangladesh. Faridpur Med Coll J 5, 14-16.

2. Kumarasinghe SPW (2000) Meteorological parameters and pattern of dermatology clinic attendance through a calendar year: a Sri Lankan experience. Int J Dermatol 39, 497-500.

3. Augustin J, Franzke N, Augustin M, Kappas M (2008) Does climate change affect the incidence of skin and allergic diseases in Germany? J Dtsch Dermatol Ges 6, 632-638.

4. Ingafou M, Leao JC, Porter SR, Scully C (2006) Oral lichen planus: a retrospective study of 690 British patients. Oral Dis 12, 463-468.

5. Ismail SB, Kumar SKS, Zain RB (2007) Oral lichen planus and lichenoid reactions: etiopathogenesis, diagnosis, management and malignant transformation. J Oral Sci 49, 89-106.

6. Pakfetrat A, Javadzadeh-Bolouri A, Basir-Shabestari S, Falaki F (2009) Oral lichen planus: a retrospective study of 420 Iranian patients. Med Oral Patol Oral Cir Bucal 14, E315-318.

7. Scully C, Beyli M, Ferreiro MC, Ficarra G, Gill Y, Griffiths $\mathrm{M}$ et al. (1998) Update on oral lichen planus: etiopathogenesis and management. Crit Rev Oral Biol Med 9, 86-122.

8. el-Zawahry M (1965) Lichen planus tropicus. Dermatol Int $4,251-254$.

9. Lundquist G, Forsgren H, Gajecki M, Emtestam L (1995) Photochemotherapy of oral lichen planus: a controlled study. Oral Surg Oral Med Oral Pathol Oral Radiol Endod 79, 554-558.

10. Boyd AS, Neldner KH (1991) Lichen planus. J Am Acad Dermatol 25, 593-619.

11. De US, Dube RK, Rao GSP (2005) Extreme weather events over India in the last 100 years. J Ind. Geophys Union 9, 173-187.

12. Mollaoglu N (2000) Oral lichen planus: a review. Br J Oral Maxillofac Surg 38, 370-377.

13. Ramírez P, Feito M, Sendagorta E, González-Beato M, De
Lucas R (2012) Childhood actinic lichen planus: successful treatment with antimalarials. Australas J Dermatol 53, e10-13.

14. Isaacson D, Turner ML, Elgart ML (1981) Summertime actinic lichenoid eruption (lichen planus actinicus). J Am Acad Dermatol 4, 404-411.

15. Kim GH, Mikkilineni R (2007) Lichen planus actinicus. Dermatol Online J 13, 13.

16. Scully C, Carrozzo M (2008) Oral mucosal disease: lichen planus. Br J Oral Maxillofac Surg 46, 15-21.

17. Rodríguez-Núñez I, Blanco-Carrión A, García AG, Rey JG (2001) Peripheral T-cell subsets in patients with reticular and atrophic-erosive oral lichen planus. Oral Surg Oral Med Oral Pathol Oral Radiol Endod 91, 180-188.

18. Wolff K, Goldsmith LA, Katz SI, Gilchrest BA, Paller AS, Leffell DJ (2008) Fitzpatrick's dermatology in general medicine. 7th ed, McGraw Hill, New York, 244-256.

19. Lacy MF, Reade PC, Hay KD (1983) Lichen planus: a theory of pathogenesis. Oral Surg Oral Med Oral Pathol 56, 521-526.

20. Girardi C, Luz C, Cherubini K, de Figueiredo MAZ, Nunes MLT, Salum FG (2011) Salivary cortisol and dehydroepiandrosterone (DHEA) levels, psychological factors in patients with oral lichen planus. Arch Oral Biol 56, 864-868.

21. Allen CM, Beck FM, Rossie KM, Kaul TJ (1986) Relation of stress and anxiety to oral lichen planus. Oral Surg Oral Med Oral Pathol 61, 44-46.

22. Christensen E, Holmstrup P, Wiberg-Jorgensen F, NeumannJensen B, Pindborg JJ (1977) Arterial blood pressure in patients with oral lichen planus. J Oral Pathol 6, 139-142.

23. Strauss RA, Fattore L, Soltani K (1989) The association of mucocutaneous lichen planus and chronic liver disease. Oral Surg Oral Med Oral Pathol 68, 406-410.

24. Al-Khenaizan S (2001) Lichen planus occurring after hepatitis B vaccination: a new case. J Am Acad Dermatol 45, 614-615.

25. Bagán JV, Ramón C, González L, Diago M, Milián MA, Cors $R$ et al. (1998) Preliminary investigation of the association of oral lichen planus and hepatitis C. Oral Surg Oral Med Oral Pathol Oral Radiol Endod 85, 532-536. 